\title{
Development of Napoleon's Theorem on the Rectangles in Case of Inside Direction
}

\author{
Mashadi $^{1,}$, Chitra Valentika ${ }^{2}$, Sri Gemawati ${ }^{1}$ \\ ${ }^{1}$ Analysis and Geometry Group Department of Mathematics, Fakulty of Mathematics and Natural Sciences University of Riau, Bina Widya \\ Campus, Pekanbaru, Indonesia \\ ${ }^{2}$ Fakulty of Mathematics and Natural Sciences, University of Riau Bina Widya Campus, Pekanbaru, Indonesia
}

Email address:

mash_mat@unri.ac.id (Mashadi), chitra.valentika@yahoo.com (C. Valentika), gemawati.sri@gmail.com (S. Gemawati)

${ }^{*}$ Corresponding author

\section{To cite this article:}

Mashadi, Chitra Valentika, Sri Gemawati. Development of Napoleon's Theorem on the Rectangles in Case of Inside Direction. International Journal of Theoretical and Applied Mathematics. Vol. 3, No. 2, 2017, pp. 54-57. doi: 10.11648/j.ijtam.20170302.11

Received: October 25, 2016; Accepted: January 12, 2017; Published: February 9, 2017

\begin{abstract}
In this paper will be discussed Napoleon's Theorem on rectangles that has two parallel pair sides of the square case that built inside direction. The theorem will be proven by using congruence approach. At the end of Napoleon's theorem was discussed the development of Geogebra application in case of inside direction.
\end{abstract}

Keywords: Napoleon's Triangle, Napoleon's Theorem on Quadrilateral, Inside Directions and Congruence

\section{Introduction}

Geogiev and Mushkarov [2, 6, 7, 10, 11 and 12] stated that Napoleon's theorem was discovered by Napoleon Bonaparte (1769-1821), a French emperor and mathematics figure in geometry. After four years he was death, the theorem was published for the first time by W. Rutherford in case of equilateral triangle that constructed in the outside direction [1, 3, 4, 6 and 7]. Then, developed by Wetzel [5, 6 and 7] in case of an equilateral triangle that constructed in the inside direction. Napoleon's theorem in direction is on each side of any triangle constructed equilateral triangle leads to the inside or outside of the third point in the center of the equilateral triangle will form a new equilateral triangle. It is called the Napoleon triangle [3, 6 and 7]. In accordance with Napoleon's Theorem on the triangle then the theorem will be developed on a rectangle. In this article the author discusses the proof of quadrilateral Napoleon's theorem is a quadrilateral that has two pairs of parallel sides such asaparallelogram, and the developement of applying Napoleon Theorem with Geo-Gebra application.

\section{Napoleon's Theorem on Triangle}

Look at picture 1 , on the $A B$ side was constructed $\triangle A B D$ equilateral triangle, and on the $\mathrm{BC}$ side was constructed $\triangle B C F$ equilateral triangle, and on the $A C$ side was constructed $\triangle A C E$ equilateral triangle, the equilateral triangles were constructed to inside direction [4, 6 and 11]. For example point $P, Q$, and $R$ are center point of equilateral triangle. The center points form equilateral triangle that can be called as inside Napoleon's triangle [4, 5, 6 and 7]. The Napoleon's theorem on the triangle in case of inside direction was provided as follow [3, 4, 7, 8 and 9].

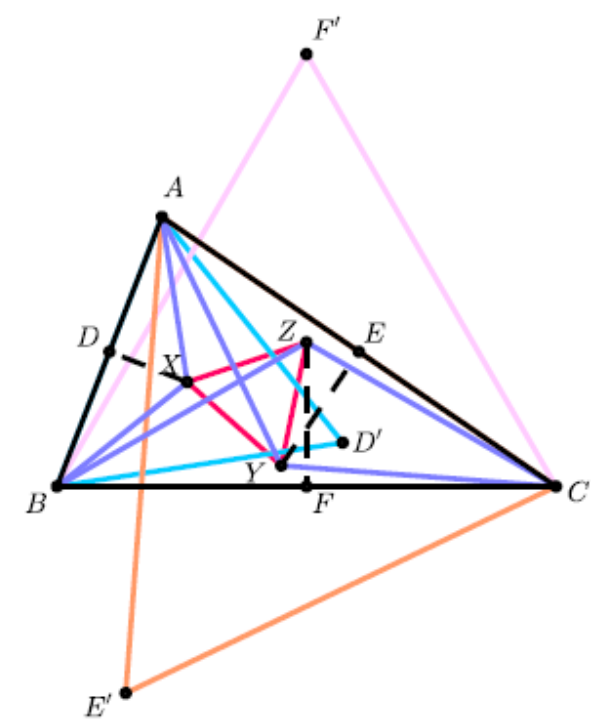

Figure 1. Napoleon's Theoremon traingle in case of inside direction. 
Theorem 1. On this case explained equilateral trianglethat constructed on each side of $\triangle A B C$ quadrilateral to inside. For example $X, Y$, and $Z$ are center point of $\triangle A B D^{\prime}, \triangle A C E^{\prime}$, and $\triangle B C F^{\prime}$, the points form equilateral triangle that can be called as Napoleon's inside triangle, the illustration is showed on figure 1.

Proof: Picture 1 is the illustration of the proof of Napoleon's theorem on the triangle that leads inside direction.

Furthermore, in providing $\triangle X Y Z$ is equilateral triangle, will be shown that $X Y=Y Z=X Z$ in accordance with trigonometry. By using basic trigonometry formula as follow

$$
\begin{aligned}
& \mathrm{BX}=\mathrm{AX}=\frac{1}{3} \sqrt{3} \mathrm{c}, \\
& \mathrm{AY}=\mathrm{CY}=\frac{1}{3} \sqrt{3} \mathrm{~b}, \\
& \mathrm{BZ}=\mathrm{CZ}=\frac{1}{3} \sqrt{3} \mathrm{a} .
\end{aligned}
$$

Then, by using cosinus directionon $\triangle B X Z, \triangle C Y Z$, and $\triangle A X Y$ as follow.

$$
\begin{gathered}
X Z^{2}=\frac{1}{3} c^{2}+\frac{1}{3} a^{2}-\frac{2}{3} a c \cos \left(60^{\circ}-\angle \mathrm{B}\right), \\
=\frac{1}{3} c^{2}+\frac{1}{3} a^{2}-\frac{2}{3} a c\left(\frac{1}{2} \cos \angle \mathrm{B}-\frac{\sqrt{3}}{2} \sin \angle \mathrm{B}\right), \\
X Z^{2}=\frac{1}{3}\left(c^{2}+a^{2}-2 a c \cos \angle \mathrm{B}\right)+\frac{1}{3} \mathrm{ac} \cos \angle \mathrm{B}+ \\
\quad \frac{\sqrt{3}}{3} \mathrm{ac} \sin \angle \mathrm{B} \\
X Z^{2}=\frac{1}{3}\left(b^{2}+a c \cos \angle \mathrm{B}\right)+\frac{\sqrt{3}}{3} a c \sin \angle \mathrm{B} \\
Y Z^{2}=\frac{1}{3} a^{2}+\frac{1}{3} b^{2}-\frac{2}{3} a b \cos \left(60^{\circ}-\angle \mathrm{C}\right) \\
=\frac{1}{3} a^{2}+\frac{1}{3} b^{2}-\frac{2}{3} a b\left(\frac{1}{2} \cos \angle \mathrm{C}-\frac{\sqrt{3}}{2} \sin \angle \mathrm{C}\right), \\
=\frac{1}{3}\left(a^{2}+b^{2}-2 a b \cos \angle \mathrm{C}\right)+\frac{1}{3} \mathrm{abcos} \angle \mathrm{C}+ \\
\quad \frac{\sqrt{3}}{3} \mathrm{absin} \angle \mathrm{C}, \\
X Y^{2}=\frac{1}{3}\left(a^{2}+b c \cos \angle \mathrm{A}\right)+\frac{\sqrt{3}}{3} \mathrm{bcsin} \angle A . \\
Y Z^{2}=\frac{1}{3}\left(c^{2}+a b \cos \angle \mathrm{C}\right)+\frac{\sqrt{3}}{3} \mathrm{absin} \angle \mathrm{C} . \\
=\frac{1}{3}\left(b^{2}+c^{2}-2 b c \cos \angle \mathrm{A}\right)+\frac{1}{3} \mathrm{bccos} \angle \mathrm{A}+ \\
{ }^{2}+\frac{1}{3} c^{2}-\frac{2}{3} b c \cos \left(60^{\circ}-\angle \mathrm{A}\right) . \\
{ }^{2}-\frac{2}{3} b c\left(\frac{1}{2} \cos -\frac{\sqrt{3}}{2} \sin \angle \mathrm{B}\right), \\
=
\end{gathered}
$$

Based on cosinus directionon $\triangle A B C$ that has been elimminated as follow

$$
\begin{aligned}
& a^{2}+b c \cos \angle \mathrm{A}=b^{2}+a c \cos \angle B, \\
& b^{2}+a c \cos \angle \mathrm{B}=c^{2}+a b \cos \angle C .
\end{aligned}
$$

Based on sinus directionon $\triangle A B C$ as follow

$$
\begin{aligned}
& \sin \angle \mathrm{B}=\frac{b}{c} \sin \angle C, \\
& \sin \angle \mathrm{A}=\frac{a}{c} \sin \angle C .
\end{aligned}
$$

Then, by distributing the equality (4) and the equality (6) to the equality (3) as follow

$$
\begin{gathered}
X Y^{2}=\frac{1}{3}\left(b^{2}+a c \cos \angle \mathrm{B}\right)+\frac{\sqrt{3}}{3} \mathrm{bc}\left(\frac{a}{c} \sin \angle C\right) \\
X Y^{2}=\frac{1}{3}\left(b^{2}+a c \cos \angle \mathrm{B}\right)+\frac{\sqrt{3}}{3} \mathrm{ab} \sin \angle C
\end{gathered}
$$

If it is distributed the equality (7) to the equality (2) as follow

$$
Y Z^{2}=\frac{1}{3}\left(b^{2}+a c \cos \angle \mathrm{B}\right)+\frac{\sqrt{3}}{3} \mathrm{ab} \sin \angle \mathrm{C} .
$$

Then by distributing the equality (7) to the equality (1) as follow

$$
\begin{aligned}
& X Z^{2}=\frac{1}{3}\left(b^{2}+a c \cos \angle \mathrm{B}\right)+\frac{\sqrt{3}}{3} \text { ac }\left(\frac{b}{c} \sin \angle B\right), \\
& X Z^{2}=\frac{1}{3}\left(b^{2}+a c \cos \angle \mathrm{B}\right)+\frac{\sqrt{3}}{3} \mathrm{absin} \angle C .
\end{aligned}
$$

Based on the equality of (8), (9) and (10) it is clear that $X Y$ $=Y Z=X Z$, so it can be inffered that $\triangle X Y Z$ is equilateral triangle.

\section{Napoleon's Theorem on the Quadrilateral}

Napoleon's theorem on the quadrilateral is discussed on the quadrilateral that has two pairs of parallel sides, one of them on the parallelogram. Look at the picture 2, on the $A B$ side is constructed $A B H G$ square, on $A D$ side is constructed $A D E F$ square, on the $C D$ side is constructed $C D K L$ square, and on the $B C$ sided is constructed $B C I J$ square. Then, each of square is constructed into inside direction. Furhtermore, each of square's center point is connected then can be called as Napoleon's inside quadrilateral.

Theorem 2. It is provided quadrilateral that form as parallelogram $A B C D$. On each of parallelogramside is constructed $A D E F, A B G H, C D K L$, and $B D K L$ squares that lead into inside. For example $M^{\prime}, N^{\prime}, O^{\prime}$, and $P^{\prime}$ are each of square centre point that constructed into inside direction. If they are connected they will form $M^{\prime} N^{\prime} O^{\prime} P^{\prime}$ square.

Proof. To showing $M^{\prime} N^{\prime} O^{\prime} P$ is square, it can be proved that $M^{\prime} N^{\prime}=N^{\prime} O^{\prime}=O^{\prime} P^{\prime}=M^{\prime} P^{\prime}$, and $\angle P^{\prime} M^{\prime} N=\angle M^{\prime} P^{\prime} O^{\prime}=90^{\circ}$. Look at picture 2, $G D$ line and $B F$ are deducted, for example deduction point is $S$ and $T$.

Then for the example $\mathrm{W}$ point is deduction point $B C$ and $A F$ line. Look at picture 2, $\angle T B S=\angle B F W, \angle F B W=\angle B T S$, then the three angles are equal $\angle F W B=\angle T S B$. Because of 
$A D E F$ square is rotated $180^{\circ}$, so the $A D E F$ square is straight with $B C I J$ so it is caused $\angle F W B=\angle T S B=90^{\circ}$. pull the line $N^{\prime} P^{\prime}$ and $M^{\prime} O^{\prime}$ so it cuts in a point, for example $R$ point. Because $F N^{\prime} / / B P^{\prime}$ then it cause $\angle T S B=\angle G V R=\angle P^{\prime} R O^{\prime}=$ $90^{\circ}$. look at $\triangle M^{\prime} A N^{\prime}$ and $\triangle O^{\prime} D N^{\prime}, M^{\prime} A=O^{\prime} D, \angle M^{\prime} A N^{\prime}=$ $\angle O^{\prime} D N^{\prime}, N^{\prime} A=N^{\prime} D$. then we obtained that $\triangle M^{\prime} A N^{\prime}$ and $\triangle O^{\prime} D N^{\prime}$ is congruence. So that $M^{\prime} N=N^{\prime} O^{\prime}$. it cause $\Delta M^{\prime} N^{\prime} O^{\prime}$ isosceles triangle so $\angle M^{\prime} N^{\prime} R=\angle O^{\prime} N^{\prime} R=45^{\circ}$. It is clear that $\angle M^{\prime} N^{\prime} O^{\prime}=\angle P^{\prime} M^{\prime} N=\angle M^{\prime} P^{\prime} O^{\prime}=90^{\circ}$. So, it is proved that quadrilateral $M^{\prime} N^{\prime} O^{\prime} P^{\prime}$ is square.

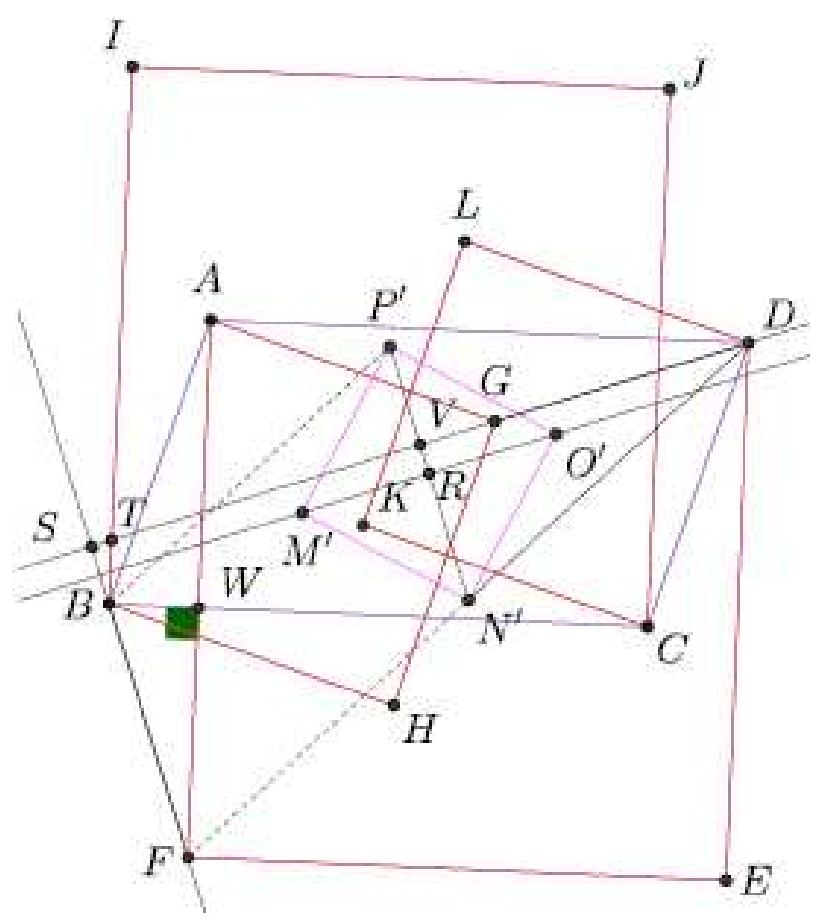

Figure 2. The Proof of Napoleon's Theorem on the Quadrilateral.

\section{Development of Napoleon's Theorem on the Quadrilateral}

Development of the quadrilateral Napoleon's theorem developed based on quadrilateral parallelogram to a square in case of leads into inside direction.

Theorem 3. Given a quadrilateral parallelogram $A B C D$, and on each side leading into the square built. Then draw a line $F G, E L, K J$, and $H I$. For example point $Q, R, S$, and $T$ is the midpoint of the fourth line. If the four points are connected, the square formed $Q R S T$.

Proof. For example point $Q, R, S$, and $T$ is the midpoint of the line FG, EL, KJ, and HI. To show QRST is square it will be proved $T Q=Q R$, and $\angle T Q R=90^{\circ}$. Figure 3, draw a line from point $Q$ to point $\mathrm{S}$ and point $\mathrm{R}$ to the point $Q$. So the lines $Q S$ and $R Q$ lines intersect at one point, said point $\mathrm{U}$. Before Show $T Q=Q R$ will at first show $U T=U R$. Figure 3, $U Y=U T, Y T=V R$ so $U T=U R$. Then Note $\triangle Q U T$ and $\triangle Q U R, \quad U T=U R, \angle T U Q=\angle T U Q$ and $U Q=U Q$ thus obtained $T Q=Q R$. From Theorem 2, $V U=U Y$, and $\angle V U Y=$ $90^{\circ}$, then also obtained $\angle Q T R=90^{\circ}$, so it proved $Q R S T$ quadrilateral is a square.

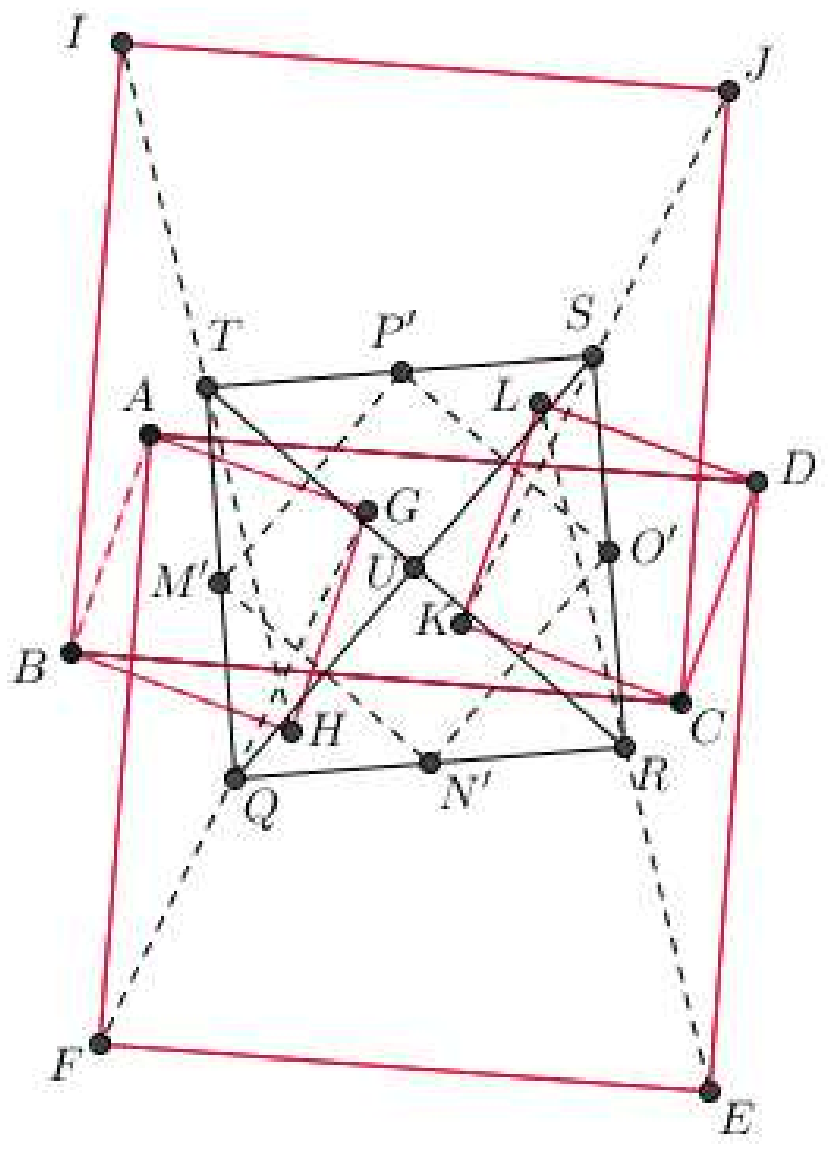

Figure 3. Development of Napoleon's theorem on the quadrilateral.

\section{Developmen of Napoleoan's Theorem with Applications Geo-Gebra}

Napoleon's Theorem development is performed by using Geogebra application. Georgiev and Mushkarov [12] stated that application Geogebra is dynamic mathematics software that can be used as a tool in the learning of mathematics. To apply Theorem Napoleon with applications GeoGebra namely by inputting equations elliptical $b x^{2}+a y^{2}=a^{2} b^{2}$ on Graphics 1 , then make four points on the graph the ellipse is to enter $\mathrm{A}=(a \cos \alpha, b \sin \alpha), \mathrm{B}=\left(a \cos \left(\alpha+90^{\circ}\right), b \sin \right.$ $\left.\left(\alpha+90^{\circ}\right)\right), \mathrm{C}=\left(a \cos \left(\alpha+180^{\circ}\right), b \sin \left(\alpha+180^{\circ}\right)\right), \mathrm{D}=(a \cos$ $\left.\left(\alpha+270^{\circ}\right), b \sin \left(\alpha+270^{\circ}\right)\right)$ on Graphics 1. for a whose value $0^{\circ}, 90^{\circ}, 180^{\circ}, 270^{\circ}$ and $360^{\circ}$ origin quadrilateral is a rhombus. Whose value for a $45^{\circ}, 135^{\circ}, 225^{\circ}$, and $315^{\circ}$ origin quadrilateral is a square or rectangular depending chart major and minor axes of the ellipse. As for the other angles of a quadrilateral origin formed is parallelogram.

Furthermore, to make the square leads into, select the Regular Polygon can be seen from the way of construction, hover the cursor back to the image and select quadrilateral. To make a point of the center of each square choose Midpoint or center can be seen from the way mengkontruksinya, hover the cursor on the second point of the square diagonal [2, h.7]. Then connect the four points of the square center, forming a quadrilateral in. Note ilutrasi Geogebra application in Figure 4. 


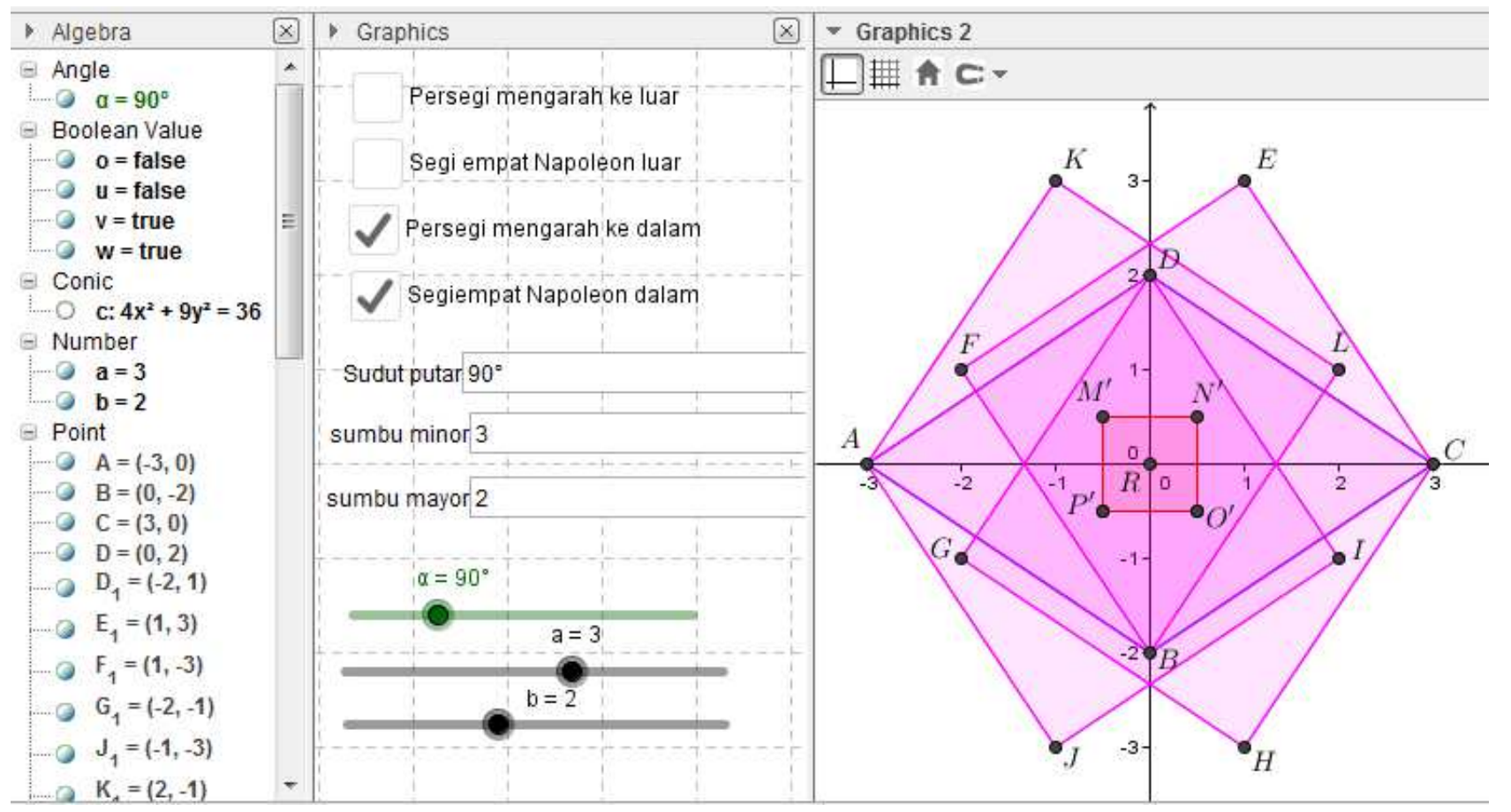

Figure 4. Napoleon's Theorem with Geogebra application.

\section{Conclusion}

After several experiments Napoleon'S theorem in the quadrilateral thus obtained Napoleon's theorem applies only to the quadrilateral which possess two pairs of parallel sides like a square, rhombus, rectangle, parallelogram. Napoleon's theorem on the line leading inside the case is if the square was built on each side, the fourth point square center will be forming a square called the Napoleon quadrilateral. Proof of that is done by using the concept of congruence. Development of Napoleon on a quadrilateral theorem can be developed to form a square on the midpoint of the line so as to form a new square.

\section{References}

[1] B. Grunbaum, Is Napoleon's theorem really Napoleon's theorem?, The American Mathematical Monthly, 119 (2012), 495-501.

[2] G. Stols, Geogebra in 10 Lessons, http://www.geogebra.org/.../GerritStols Geogebrain10Lesson.

[3] G. A. Venema, Exploring advanced euclidean geometry with Geometer's Sketchpad,

http://www.math.buffalostate.edu/giambrtm/MAT521/eeg.pdf.

[4] J. A. Abed, A proof of Napoleon's theorem, The General Science Journal, (2009), 1-4.
[5] J. E. Wetzel, Converses of Napoleon's theorem, The American Mathematical Monthly, 99 (1992), 339-351.

[6] Mashadi, Buku Ajar Geometri, Pusbangdik Universitas Riau, Pekanbaru, 2012.

[7] Mashadi, Geometri Lanjut, Pusbangdik Universitas Riau, Pekanbaru, 2015.

[8] Mashadi, S. Gemawati, Hasriati and H. Herlinawati, Semi excircle of quadrilateral, JP Journal. Math. Sci. 15 (1 \& 2) (2015), 1-13.

[9] Mashadi, S. Gemawati, Hasriati and P. Januarti, Some result on excircle of quadrilateral, JP Journal Math. Sci. 14 (1 \& 2) (2015), 41-56.

[10] M. Corral, Trigonometry, http://www.biomech.uottawa.ca/fran09/enseignement/notes/ trgbook.pdf.

[11] P. Bredehoft, Special Cases of Napoleon Triangles, Disertasi Master of Science, University of Central Missouri, 2014.

[12] V. Georgiev and O. Mushkarov, Around Napoleon's theorem, http://www.dynamat.v3d.sk/ uploadpdf/2012‘0221528150.pdf.

[13] Zukrianto, Mashadi and S Gemawati, A Nonconvex Quadrilateran and Semi-Gergonne Points on it: Some Results and Analysis, Fundamental Journal of Mathematics and Mathematical Sciences, 6 (2), 2016, 111-124. 